\title{
The Effect of Antiepileptic Therapy on the Development of Cognitive Functions in Children with Epilepsy: Introduction To The Problem
}

${ }^{1,3}$ Rimma Gamirova, ${ }^{2}$ Tatyana Akhutina, ${ }^{4}$ Elena Gorobets,${ }^{* 5}$ Ruzalina Shaykhutdinova

${ }^{1,4,5}$ Kazan Federal University, Russia, ${ }^{2}$ Moscow State University (Moscow, Russia), ${ }^{3}$ Kazan State Medical Academy, Russia, A.Yu.Ratner Pediatic Hospital №8 (Kazan, Russia)

*Email: elena_gorobets@mail.ru

\section{Received: 15th December 2017, Accepted: 20th December 2017, Published: 31st December 2017}

\begin{abstract}
Epilepsy is a chronic disease, and the control of seizures requires a long-term - and sometimes lifelong - treatment with antiepileptic drugs (AEDs). The most important goal of epilepsy pharmacotherapy is the maximum effectiveness of AEDs, which is determined by the ability to completely stop epileptic seizures with a minimum of side effects. The most frequent undesirable drug reactions recorded during antiepileptic therapy are their sedative effect, memory impairment and attention problems. In this regard, it is especially important to assess the impact of AEDs on cognitive functions during the long-term treatment. The article is devoted to the problem of studying the development of speech and other cognitive functions in children with different forms of epilepsy against the background of antiepileptic therapy using a set of methods for studying brain processes. The number of children with speech disorders and the delay in the development of cognitive functions in the world is steadily increasing. A special group of children with disorders of this kind are children with epilepsy, since they are contraindicated with drugs traditionally used by neurologists in the therapy of speech disorders. The influence of antiepileptic therapy on the development of speech functions at the moment has not been studied in detail. The linguistic component of psychometric instruments, including the evaluation of speech development, cognitive functions, especially in children and adolescents with epilepsy in Russia currently needs serious scientific development and experimental justification with a focus on European samples with the aim of optimizing joint international research.
\end{abstract}

Keywords: Epilepsy, Cognitive Functions, Assessment, Antiepileptic Drugs, Clinical Linguistics, Developmental Disorders

\section{Introduction}

The complexity of assessing the effect of AEDs on cognitive functions in persons with epilepsy is that the state of cognitive functions may also depend on several factors, among which the side effects of AEDs are only one of many assumed causes. In connection with this, the most difficult problem is the use of an adequate methodology that makes it possible to isolate and evaluate the side effect of AEDs. One way to compare the effect of AEDs on cognitive functions may be to test patients with newly diagnosed epilepsy before and during treatment at optimal therapeutic doses in a randomized, double-blind study [Perucca 2007, 3: 194].

The effect on speech is not usually emphasized in the list of side effects of antiepileptic drugs. For the first time, the negative effect on speech was first mentioned in the study of undesirable drug reactions of topiramate. In patients who received topiramate, amnestic aphasia and difficulties in word selection were reported [Ojemann 2001, 2: 581]. It should be noted that mainly adult patients were included in the studies, while there are practically no such publications for children.

Neuropsychological and neurolinguistic diagnostics of children with epilepsy is also specific. Existing methods for assessing the development of higher mental functions take a long time and require additional skills, whereas in the case of epilepsy, therapy should be prescribed immediately and the neurologist has to make the fastest diagnosis.

\section{Materials and Methods \\ The research was conducted in the laboratory "Clinical Linguistics" (Kazan Federal University) and at A.Yu.Ratner Pediatic Clinical Hospital №8 (Kazan, Russia).}

The methodological principles are aimed at the development of personalized medicine, within the framework of which the study of higher cortical functions of a person at different stages of his life is one of the main tasks. It is essential to use the achievements of neuropsychology, clinical and cognitive linguistics to develop a linguistically valid diagnostic tool used in conjunction with functional diagnostics (continued video EEG monitoring with sleep included) and neurological examination, especially for children with different forms of epilepsy [Gamirova 2017a] and epileptiform activity [Gamirova 2017b].

The epilepsy is revealed with the help of electroencephalographic study according to a standard procedure using the international electrode arrangement "10-20" in bipolar installation. The recording of the electroencephalogram (EEG) is performed in wakefulness and daytime sleep on the 19-channel computer electroencephalograph EEG- 
A-21/26 Encephalan-131 M (research and production company Medikom-MTD, Taganrog).

\section{Results}

Cognitive impairment is quite common in patients with epilepsy and causes a lot of serious problem. According to the results of the research, $44 \%$ of patients with epilepsy report difficulties in learning, $45 \%$ notice the negative thinking in the speed and quality of thinking, $63 \%$ of patients believe that the side effects of antiepileptic drugs (AEDs) do not allow them to fully realize themselves in life [Meador 2006, 68: 63]. AEDs affect the functions of cognition, which is due to their mechanism of action on the central nervous system.

The cognitive functions of patients with epilepsy can be influenced by: 1) the form of epilepsy, 2) the age of the onset of epilepsy, 3) the possibility to stop the attacks, 4) the index of epileptic activity (there can be no seizures in the patient, while the epileptiform activity functionally blocking the corresponding centers of the brain influences negatively, 5) side effects of antiepileptic drugs, 6) comorbid organic background (for example, degenerative neurological diseases, hereditary metabolic disorders, etc., in which epilepsy is one of the symptoms of the disease).

Despite the fact that the degree of side effects on cognitive functions is usually considered moderate for most AEDs, their effect can be significant, especially in childhood, when it comes to the impact on the development of speech functions and learning. In addition, side effects can "accumulate" with prolonged therapy, which affects the daily life of patients, especially with refractory epilepsy.

The main mechanism that determines the negative effects of AEDs on cognitive functions is related to the general effect of antiepileptic drugs on neuronal membranes and neurotransmission. In general, the antiepileptic mechanism of drugs manifests either in suppressing neuronal excitability, or in enhancing inhibition of neurons.

The mechanisms of the influence of AEDs with GABA-ergic properties on cognitive functions have been previously studied. Traditionally, older AEDs, such as barbituric acid preparations, phenytoin, are thought to adversely affect a wide range of cognitive functions such as attention, memory, and reaction times [Smith 1975, 23: 208; Thompson 1980; 80: 76; Thompson 1981, 20: 159; Meador 1991, 41: 1538; Meador 1993, 34: 156].

\section{Discussion}

In foreign and Russian practice, the following diagnostic batteries are traditionally used:

A) The Halstead-Reitan Neuropsychological Test Battery (HRNB) - the battery with quantitative assessment, which has two variants for children. It includes a series of tests: Tactual Performance Test; Seashore Rhythm Test; Speech Sounds Perception Test; Finger Tapping Test; Sensory Perceptual Examination etc. [Reitan 1985]. This battery is not aimed at a detailed analysis of speech processes and does not take into account the age specificity to a proper degree.

B) Luria-Nebraska Neuropsychological Battery for children (LNNB-C) is a standardized test that identifies neuropsychological deficiencies by measuring functioning on fourteen scales (motor, rhythm, tactile, visual, receptive speech, expressive speech, writing, reading, arithmetic, memory, intellectual processes, pathognomonic, left hemisphere, and right hemisphere).

C) The battery of neuropsychological examination of children from 6 to 9 year old (T. Akhutina et al.) [The methods 2016]. This battery can be used to evaluate children aged 6-9 years, and it did not have a purpose to assess all language levels. The battery assesses three functional blocks of the brain: a block of programming, regulation and control; a block of receiving, storing and processing information; power block. For each of the blocks there was developed series of tests.

Also the batteries that evaluate the level of intelligence are actively used: Wechsler Adult Intelligence Scale (WAIS) [Kaufman 2006], which estimates the level of nonverbal intelligence and erudition, Raven's Progressive Matrices [Raven 1981] which estimate the level of nonverbal intelligence. WAIS can hardly be used in clinical practice, especially for the estimation of children with epilepsy, and many questions in it are built on erudition. Raven's matrices make it possible to assess the safety of primary intelligence (although the multiplicity of this assessment raises questions).

Specific speech tests are presented by the MacArthur questionnaire [Fenson 1993] (qualitativequantitative, standardized, adapted in a Russianspeaking environment, but intended for children from 8 months to 3 years old and therefore not suitable for use the assessment of children of other age), as well as speech cards and schemes developed by speech therapists: Filicheva T.B., Chirkina G.V. Programs of preschool educational institutions of compensatory type for children with disturbances of rechy; Balobanova V.P., Titova T.A., Chistovich I.A. Diagnosis of speech disorders in children and the organization of speech therapy work in the conditions of a pre-school educational institution; Nishcheva N.V. Speech cards for children 3-4 and 57 years old. These schemes are not applicable in the 
research as they do not contain a quantitative estimate.

Thus, it is necessary to create a tool for rapid assessment of the neuropsychological and speech status with the possibility of quantitative calculation of the results, which would be ranked at ages from four to eighteen years and could be used by neurologists up to initiation of therapy and subsequently used to assess changes in the cognitive status during the courses of AEDs therapy.

\section{Conclusions}

In comparative evaluation of the negative impact of different AEDs on the cognitive functions in children and adolescents with epilepsy, the most promising are the studies which include the use of specially developed neuro psychologically and linguistically validated tests to assess cognitive functions and speech status for children and adolescents suffering from idiopathic forms of epilepsy ranked for different ages before treatment and further in dynamics during the AEDs therapy.

The linguistic component of psychometric instruments, including the evaluation of speech development, cognitive functions, especially in children and adolescents with epilepsy - one of the chronic, often disabling diseases, in Russia currently needs serious scientific development and experimental justification with a focus on European samples with the aim of optimizing joint international research.

None of the existing methods of cognitive functions assessment gives a possibility of express evaluation that could be used in clinical practice in a "quick response" situation (in the event of epileptic seizures, therapy should be administered immediately, and the cognitive functions of patients need to be evaluated very quickly).

Evaluation of the isolated effect of AEDs impact on cognitive functions is probably most acceptable in children with idiopathic forms of epilepsy, in which, unlike age-dependent epileptic encephalopathies, the effect of epilepsy itself on cognitive functions is minimal.

\section{Conflict of Interest}

The authors confirm that the data presented do not contain conflict of interest.

\section{Acknowledgements}

The work is performed according to the Russian Government Program of Competitive Growth of Kazan Federal University.

\section{References}

1. Gamirova R.G., Farnosova M., Zaykova F.M. A Cortisol Level during the Adrenocorticotropic Hormone Treatment of West Syndrome Patients as a Potential Prognostic Factor for Cessation of Infantile Spasms. Annals of Neurology. - 2017. T.82, Suppl. 21. SI. - P. 64.

2. Gamirova R.G., Gorobets E.A., Marulina V.I., Bolshakova T.A., Zaykova F.M. Developmental disorders in children with cognitive epileptiform disintegration. 4th International Scienfific Conference on Social Science \& Arts SGEM 2017, 3(II): Psychology and Psychiatry, Language and Linguistics, 2017. - Pp. 107-114.

3. Eliseeva M.B., Vershinina E.A., Ryskina V.L. The MacArthur Inventories: Russian version. Assessment of speech and communication development in children of early age. The norms of development. The samples of analysis. Comments. - Ivanovo: LISTOS, 2016. 76 p.

4. Fenson, L., Dale, P.S., Reznick, J.S., Thal, D., Bates, E., Hartung, J.P., Pethick, S., \& Reilly, J.S. The MacArthur Communicative Development Inventories: User's Guide and Technical Manual. - Baltimore: Paul H. Brokes Publishing Co, 1993.

5. Kaufman A.S., Lichtenberger, E. (2006). Assessing Adolescent and Adult Intelligence (3rd ed.). - Hoboken (NJ): Wiley, 2006.

6. Meador K.J. et al. Comparative cognitive effects of carbamazepine and phenytoin in healthy adults. Neurology. - 1991. - V.41. - P.1537.

7. Meador K.J., Loring D.W., Allen M.E. et al. Effects of carbamazepine and phenytoin on EEG and memory in healthy adults. Epilepsia. - 1993. - V.34. - P.153-157.

8. Meador K.J. Cognitive and memory effects of the new antiepileptic drugs. Epilepsy Res. - 2006. - V.68. - P.63-67.

9. Ojemann L. Language Disturbances as Side Effects of Topiramate and Zonisamide Therapy. Epilepsy \& Behavior. - 2001. Vol. 2. - Pp. 579-584.

10. Perucca E. Withdrawing antiepileptic drugs in seizure-free patients: what are the cognitive benefits? Nat Clin Pract Neurol. 2007. - V.3. - P.194-195.

11. Raven, J. Manual for Raven's Progressive Matrices and Vocabulary Scales. Research Supplement No.1: The 1979 British Standardisation of the Standard Progressive Matrices and Mill Hill Vocabulary Scales, Together With Comparative Data From Earlier Studies in the UK, US, Canada, 
Germany and Ireland. San Antonio, Texas: Harcourt Assessment, 1981.

12. Reitan, R.M. Halstead-Reitan Neuropsychological Test Battery: Theory and Clinical Interpretation. - Tucson, Arizona: Reitan Neuropsychology, 1985.

13. Smith W.L., Lowrey J.B. Effects of diphenylhydantoin on mental abilities in the elderly. J Am Geriatr Soc. - 1975. - V. 23. - P.207-211.

14. The methods of neuropsychological examination of children from 6 to 9 year old / Ed. by T.V.Akhutina. Moscow: V.Sekachev, 2016. 280 p.

15. Thompson P. Anticonvulsant drugs, cognitive function and memory / P.Thompson, F.Huppert, M.Trimble // Acta Neurol Scand. - 1980. - V. 80. - P. 75-81.

16. Thompson P., Huppert F.A., Trimble M. Phenytoin and cognitive function: effects on normal volunteers and implications for epilepsy. Br J Clin Psychol. - 1981. - V.20. -P.155-162. 